\title{
Identification of Contrast Induced Nephropathy (CIN) with intravenous iso-Osmolar contrast media in renal failure patients with and without additional comorbidities undergoing Computed Tomography
}

\author{
Thomas Mathew ${ }^{1}$, Dr Prakashini K ${ }^{2}$, Rahul P Kotian ${ }^{3 *}$ \\ ${ }^{1}$ Mr. Thomas Mathew, MSc Medical Imaging Technology, School of Allied Health Sciences, Manipal University, \\ Manipal, ${ }^{2}$ Dr Prakashini K, Professor, Department of Radiodiagnosis and Imaging, Kasturba Medical College, Manipal, \\ Manipal University, ${ }^{3}$ Mr. Rahul P Kotian, Senior Scale Assistant Professor, MSc. Medical Imaging Technology, \\ Department of Medical Imaging Technology, Manipal School of Allied Health Sciences, Manipal University, Manipal, \\ Karnataka, India
}

Address for Correspondence: Dr. Prakashini K, Professor, Department of Radiodiagnosis and Imaging, Kasturba Medical College, Manipal, Manipal University, Karnataka, India. E-mail: docprakashinik@ yahoo.co.in

\begin{abstract}
Aim: To identify contrast induced nephropathy with intravenous iso-osmolar contrast media in renal failure patients with additional comorbidities undergoing computed tomography. Study design: Hospital based observational study. Methods: A retrospective study was conducted on 207 patients with pre-existing renal failure and comorbidities. In this study we collected pre-contrast creatinine values available within 3 weeks and post-contrast creatinine value was collected on 2nd and 5th day following CM injection in order to check early and late contrast induced nephropathy (CIN). Rate of CIN with and without comorbid conditions in CKD patients were calculated. Then we assessed the incidence of CIN with different amount of CM administered. We also calculated the relation of CIN with pre-contrast creatinine and eGFR range, which was categorized as $1.4-2.0 \mathrm{mg} / \mathrm{dL}, 2.0-3.0 \mathrm{mg} / \mathrm{dL}$ and $>3 \mathrm{mg} / \mathrm{dL}$ and $>60 \mathrm{ml}$ and $<60$ $\mathrm{mL} / \mathrm{min}$ per $1.73 \mathrm{~m} 2$ respectively. Results: Rate of CIN was relatively higher in renal failure patients and also in patients with comorbidities especially diabetes mellitus. Risk of CIN development was possible on $5^{\text {th }}$ day also which was considered as delayed CIN. It was shown in the study that as the amount of contras media increases, rate of CIN also increases which was prominent in case of patients administered with $>80 \mathrm{ml}$ of contrast media. CIN risk was better determined by eGFR than Creatinine value. Conclusion: CIN rate was clinically significant in CKD patients with comorbidities who received an iso-osmolar contrast media.
\end{abstract}

Keywords: CIN, Contrast Induced Nephropathy; IOCM, Iso-osmolar Contrast Media; SCr, Serum Creatinine

\section{Introduction}

The contrast enhanced CT scan is very helpful for accurate clinical staging of metastatic lesions of parts such as breast and liver and it's extensions to other parts of body [1]. However Contrast induced nephropathy (CIN) is one of major adverse reaction associated with iodinated contrast media and which can even result in renal impairment and hospitalization [2]. The occurrence of contrast induced nephropathy (CIN), also

Manuscript received $10^{\text {th }}$ September 2016

Reviewed: $20^{\text {th }}$ September 2016

Author Corrected: $4^{\text {th }}$ October 2016

Accepted for Publication 17 $7^{\text {th }}$ October 2016 referred to as acute kidney injury due to contrast media is a substantial complication of intravascular contrast medium (CM) use which is linked with excess morbidity and mortality. Even though exact pathophysiology is not clear CIN is considered to be acute disease condition of renal tubules due to toxicity of contrast media or due to renal hemodynamic changes. Generally kidney function is measured by indicators such as serum creatinine ( $\mathrm{SCr}$ ), estimated glomerular filtration rate (eGFR) and creatinine clearance $(\mathrm{CrCl})$. Contrast induced nephropathy is 
defined as rise in serum creatinine above $0.5 \mathrm{mg} / \mathrm{dL}$ or a rise of $25 \%$ from base line or reduction in eGFR to $<60$ $\mathrm{mL} / \mathrm{min}$ per $1.73 \mathrm{~m} 2$ within 2 to 3 days following CM administration:[4]. The increased amount of CM, an elderly population and rise in chronic kidney disease (CKD) can result in increased incidence of CIN unless effective preventative measures are used. The major risk factor predicting $\mathrm{CIN}$ is pre-existing $\mathrm{CKD}$, which can be predicted from glomerular filtration rate (GFR) or Serum creatinine values. Contrast media with lowosmolality will reduce the adverse incidence. DM, chronic renal failure $(\mathrm{SCr}>1.5 \mathrm{mg} / \mathrm{dL})$, congestive heart failure and large volume of CM $(>70 \mathrm{ml})$ are considered as risk factors for contrast induced nephropathy. In previous studies, CKD and diabetes individually and together were considered as a risk factors for CIN [3].

In the present study we have evaluated the incidence of CIN in patients who were already having renal failure defined as the pre-contrast creatinine values greater than $1.4 \mathrm{mg} / \mathrm{dL}$. We have also derived an incidence and Odds Ratio for additional co morbidities like Diabetis, hypertension and sepsis in preexisting CKD patients.

\section{Materials and Methods}

Hospital based observational study was performed on patients who were referred for contrast enhanced computed tomography between March 2014 to December 2015.The Inclusion criteria included, patients with pre-existing chronic renal failure, having $\mathrm{SCr}$ value above the normal limit of $1.4 \mathrm{mg} / \mathrm{dl}$. Patients who were referred by clinician for contrast enhanced CT scan study of any part of the body for specific indications were included.

The patient was excluded if pre-creatinine values were not available within 3 weeks in case of stable patients and within a week in case of unstable patients or in acute renal injury. Patients whose post creatinine values were not available within a week time were also excluded.
Study procedure- The study approval was provided by the institutional research committee. Considering the inclusion and exclusion criteria 207 patients were included with an age range of 20-80 yrs. The samples selected were a population undergoing CECT scan with iso-osmolar contrast media (IOCM) iodixanol with precontrast creatinine value equal or greater than 1.4 $\mathrm{mg} / \mathrm{dL}$.

Contrast induced nephropathy (CIN) was defined as either greater than $25 \%$ increase of serum creatinine or an absolute increase in SCr of $0.5 \mathrm{mg} / \mathrm{dL}$ from baseline after intravascular administration of contrast media. ${ }^{[4]}$ Post-contrast creatinine values were collected on 2nd and 5th day following $\mathrm{CM}$ injection in order to check early and late contrast induced nephropathy (CIN). Detailed history was taken from the case files. Amount of CM used for the scan and co-morbid conditions like HTN, DM, sepsis and dehydration status of the patients were documented.

Statistical Analysis- The collected data was entered into SPSS after which the incidence of CIN on 2nd day and 5th day was separately calculated. Later to know the overall incidence, we categorized it into "CIN present" when there was rise in serum creatinine by 0.5 $\mathrm{mg} / \mathrm{dl}$ either on 2 nd or 5th day. Rate of CIN with and without comorbid conditions in CKD patients was calculated.

Then we assessed the relation of CIN with different amount of CM administered. We also calculated the relation of CIN with pre-contrast creatinine range, which was categorized as $1.4-2.0 \mathrm{mg} / \mathrm{dL}, 2.0-3.0$ $\mathrm{mg} / \mathrm{dL}$ and $>3 \mathrm{mg} / \mathrm{dL}$ and eGFR range.

Thereafter the odds ratio was calculated to assess whether the incidence of CIN was statistically significant and to check the relationship between comorbidities and incidence of CIN.

\section{Results}

In the present study out of 207 patients with pre-existing renal failure ( $\mathrm{SCr}>1.4 \mathrm{mg} / \mathrm{dL}$ ), 35 of them developed CIN either on 2 nd or on $5^{\text {th }}$ day. The incidence of 'early' CIN (2nd day) was 13 and 'late' CIN (5th day) was 14. Out of 207 patients 136 patients had comorbidities. Most common associated comorbid conditions were HTN, both DM and HTN together, DM alone followed by other conditions like sepsis, AKI (Acute kidney injury) and dehydration in combination with DM and HTN or alone. Out of 136 CKD patients having other comorbidities 29 developed CIN, whereas 71 patients without any comorbidity, 6 developed CIN. Rate of CIN was relatively more in patients with diabetes mellitus and HTN in combination (Table 1). Rest of the comorbidities also induced CIN however number of other comorbidities were less, constituting 37 in number. Out of 37 cases 11 developed CIN. 
Table-1:- Descriptive analyses of comorbidities vs. 2nd or 5th day post contrast CIN status

\begin{tabular}{|c|c|c|c|}
\hline \multirow[t]{2}{*}{ Co morbidities } & \multicolumn{2}{|c|}{ Post contrast creatinine on $2^{\text {nd }}$ or $5^{\text {th }}$ day } & \multirow[t]{2}{*}{ Total } \\
\hline & CIN Present & CIN Absent & \\
\hline No comorbidities & 6 & 65 & 71 \\
\hline HTN & 4 & 38 & 42 \\
\hline Diabetes & 2 & 15 & 17 \\
\hline Diabetes \& HTN & 12 & 28 & 40 \\
\hline Sepsis & 2 & 5 & 7 \\
\hline Dehydration & 0 & 5 & 5 \\
\hline Sepsis, DM \& HTN & 2 & 2 & 4 \\
\hline AKI & 1 & 4 & 5 \\
\hline AKI DM \& HTN & 2 & 3 & 5 \\
\hline HTN \& AKI & 2 & 6 & 8 \\
\hline DM \& Sepsis & 2 & 0 & 2 \\
\hline HTN \& Sepsis & 0 & 1 & 1 \\
\hline Total & 35 & 172 & 207 \\
\hline
\end{tabular}

Predominant number of patients received $80 \mathrm{ml}$ of contrast media. It is shown in the table 2 , that as the amount of contrast increases rate of CIN also increases. Out of 150 patients who were administered with 80 ml of iso-osmolar contrast media 30 developed CIN whereas 50 patients who received 40 to $60 \mathrm{ml}$ of contrast, only 3 developed CIN.

Table-2: Cross tabular analysis of amount of contrast media with incidence of CIN on either $2 \mathrm{nd}$ or 5 th day

\begin{tabular}{|c|c|c|c|}
\hline \multirow{2}{*}{$\begin{array}{c}\text { Amount of Contrast } \\
\text { Media(ml) }\end{array}$} & \multicolumn{2}{|c|}{ Total } \\
\cline { 2 - 4 } & CIN Present & 120 & 150 \\
\hline 80 & 30 & 37 & 40 \\
\hline 60 & 3 & 7 & 7 \\
\hline 40 & 0 & 3 & 4 \\
\hline 100 & 1 & 2 & 3 \\
\hline 120 & 1 & 3 & 3 \\
\hline 50 & 0 & $\mathbf{1 7 2}$ & $\mathbf{2 0 7}$ \\
\hline Total & $\mathbf{3 5}$ & & \\
\hline
\end{tabular}

Though we have not evaluated CIN only on $2^{\text {nd }}$ day or $5^{\text {th }}$ day, there was significant increase incidence of CIN when we consider either $2^{\text {nd }}$ or $5^{\text {th }}$ day rate with respect to overall incidence, or its association with comorbidities and amount of contrast media used.

We further categorized the creatinine values in to different range as shown in Table- 3 to derive the relation between range of creatinine and CIN incidence.

Table-3: Descriptive analysis of Pre-contrast creatinine range vs. CIN on 2nd or 5th day

\begin{tabular}{|c|c|c|c|}
\hline \multirow{2}{*}{$\begin{array}{c}\text { Pre-contrast Creatinine } \\
\text { Range }\end{array}$} & \multicolumn{2}{|c|}{$2^{\text {nd }}$ or $5^{\text {th }}$ day } & \\
\hline & CIN Present & CIN Absent & \\
\hline $1.4-2.0 \mathrm{mg} / \mathrm{dL}$ & 27 & 134 & 161 \\
\hline $2.0-3.0 \mathrm{mg} / \mathrm{dL}$ & 4 & 23 & 27 \\
\hline$>3 \mathrm{mg} / \mathrm{dL}$ & 4 & 15 & 19 \\
\hline Total & 35 & 172 & 207 \\
\hline
\end{tabular}

According to our result, CIN rate was high in patients with creatinine range of 1.4 to $2.0 \mathrm{mg} / \mathrm{dl}$ compared to creatinine of $>2 \mathrm{mg} / \mathrm{dl}$. Total 161 patients were having creatinine in the range of 1.4 to $2.0 \mathrm{mg} / \mathrm{dL}$ and 27 patients developed CIN, 
which was significantly more compared to creatinine $>2 \mathrm{mg} / \mathrm{dl}$. Out of 161 cases, 96 patients had co existing comorbid conditions also. This could be attributed to, difference in no of patients in this category of creatinine or because of coexisting comorbidities. And also patients with higher rate of creatinine usually undergo dialysis /hydration therapy following contrast administration which might apparently alter the result. Out of 34 patients who developed CIN, 13 were having GFR in the range of $45-59 \mathrm{ml} / \mathrm{min}$ falling in to low risk group, 15 were in moderate risk group with a GFR ranging from 31-45ml/min and another 6 were in high risk group with GFR $<30 \mathrm{ml} / \mathrm{min}$. (Table 4).

Table 4: Correlation of eGFR and CIN

\begin{tabular}{|c|c|c|c|}
\hline $\begin{array}{c}\text { Pre-contrast eGFR } \\
\text { Range }\end{array}$ & 2nd or 5th day & \multirow{2}{*}{ Total } \\
\cline { 2 - 4 } & CIN Present & CIN Absent & 12 \\
\hline$>59$ & 0 & 12 & 56 \\
\hline $45-59$ & 13 & 43 & 98 \\
\hline $31-45$ & 15 & 83 & 41 \\
\hline$<30$ & 7 & 34 & $\mathbf{2 0 7}$ \\
\hline Total & $\mathbf{3 5}$ & $\mathbf{1 7 2}$ & \\
\hline
\end{tabular}

\section{Discussion}

Development of CIN is influenced by osmolality, ionicity and the molecular structure of contrast media. $\mathrm{CM}$ acts on different anatomical sites of kidney and causes damage by various mechanisms. It damages renal tubular cells, causes vasoconstriction, osmotic property of $\mathrm{CM}$ reduces water reabsorption causing buildup of interstitial pressure, by increasing blood viscosity and decreasing red cell deformability which increases resistance to blood flow. All these mechanisms cause renal ischemia and tubular damage at cellular level.

The present study was done on 207 patients with CKD having comorbid conditions such as DM, HTN and sepsis. The major role of present study was to find the incidence of CIN in patients with pre-existing renal failure also having comorbid conditions such as diabetics and hypertension. It is already documented by previous studies that CIN occurs with both iso and low osmolar contrast media and especially in patients with comorbidities like chronic kidney disease, diabetes, hypertension and other comorbid conditions like sepsis and hydration status. However there are no results or studies performed on Indian population. Also previous all studies were given a comparative CIN rate between iso-osmolar and low-osmolar contrast media on intra arterial use especially coronary angiography studies[4,5,9].

In our study the overall rate of CIN observed was $17 \%$. According to various previous studies CIN rate varied from 1.4 to $12.4 \%$. However number of patients included in previous studies was variable. The study by
Solomon et al included 210 patients with CIN rate of $12.4 \%$ which was close to our study [6]. The studies conducted by McCullough et al, Kuhn et al, Nguyen et al and Thomson et al exhibited CIN rate with varying sample sizes of $1.4 \%(138), 5.6 \%(123), 8.5 \%(61)$ and $4.7 \%(148)$ respectively[3,7,8,9]. It was found that the rate of CIN in diabetic patients was $11.7 \%$ and was increased to $30 \%$ when having both DM and HTN together in our study. The diabetes and hypertension were found to be significant risk factor with Odds Ratio being more than one. Even though patients with both DM and HTN together were found to be clinically significant with CIN rate of $30 \%$ however showed no statistical association (OR-0.373) in developing CIN.

According to previous studies, CKD patients with either DM or HTN were showing higher rate of CIN, more so in patients with DM and HTN together. Our study also showed similar result that is CKD patients with DM or HTN higher rate of CIN (29/136) when compared to CKD without co-morbidities (6/71) constituting $21 \%$ and $8.4 \%$ respectively. In this study we also found that the amount of iso-osmolar contrast media of $80 \mathrm{ml}$ and above can be an independent risk factor for CIN (OR 4.01) when compared to patients administered with 40$60 \mathrm{ml}$ of contrast media.

Previous study by Kuhn et al stated that as renal function worsens the risk of CIN increases. However we did not find any similar result in our study. This might be because of large number of patients were in the milder grade of renal failure with creatinine value ranging from 1.4-2.0 mg/dL [7]. 
The main strength of our study was that we calculated the incidence of CIN on 2nd and 5th day following contrast media injection and found that it was $10 \%$ and $10.6 \%$ respectively where as in previous studies they followed the incidence of CIN only till 3rd day [8]. We also found that few patients developed CIN only on 5th day (delayed CIN). No previous studies have evaluated the rate of CIN on 2nd and 5th day separately to know the rate of late CIN. We have studied post contrast CIN rate on 2nd and 5th day and it was found that there were significant number of cases which developed CIN on 5th day indicating that, delayed CIN is also possible complication, which also needs attention especially in group of patients at risk. Though most of the CIN are transient, the timely and adequate management plays a main role during acute phase. Hence all high risk patients undergoing contrast study should be checked for post contrast serum creatinine for 5 to 7 days.

All studies done till now by various authors were comparative studies that were based on the nephrotoxicity of IOCM with respect to LOCM. The studies done by Solomon et al, Kuhn et al and pooled study done by Heinrich et al state that both LOCM and IOCM shows no significant difference in incidence of CIN even with pre-existing co-morbid conditions $[6,7,10]$. The study done by Heinrich et al also states that in patient with renal insufficiency a reduction in incidence of CIN was observed with IOCM through intra-arterial route of administration [10]. The studies done by McCullough et al, Nguyen et al, Ho Jo et al and Hernandez et al found that IOCM caused less nephrotoxicity than LOCM and all of them studied the effect of adverse morbid conditions on incidence of CIN $[3,4,8,11]$. With an exception compared to all other previous study results, the study done by Thomson et al stated that the IOCM caused higher rate of CIN than LOCM in patients with renal failure [9].

Most of the previous investigators had studied the effects of DM in developing CIN in patients with preexisting renal failure. In a study conducted by Kuhn et al and Solomon et al suggested that the rate of CIN in patients with diabetes and chronic kidney disease receiving IV contrast medium was not significantly different between LOCM and IOCM [6,7]. A metaanalysis conducted by McCullough et al and Nguyen et al et al states that iso-osmolar contrast media contain lower rate of developing CIN especially in case of patients with pre-existing chronic renal disease or with CKD with DM. Study also states that IOCM is associated with less rise in SCr levels as compared to LOCM [3,8]. The studies conducted by Solomon et al, McCullough and Kuhn et al found that the comorbidities such as CKD and DM has no relationship with incidence of CIN which was opposing the results found by the present study[3,6,7]. The previous studies the authors Kuhn et al and Ho Jo et al studied the effect of amount of CM with the incidence of CIN and they found that even the amount of CM $140 \mathrm{ml}$ and above is not associated with increase in SCr levels [4,7]. This was against present study results and found that the amount of contrast media administered $80 \mathrm{ml}$ and above was an independent risk factor for CIN. This is probably because our study population included CKD with comorbidities whereas Kuhn et al and Ho Jo et al included normal patients.

Isoosmolar contrast media can be advisable up to creatinine level of $1.9 \mathrm{mg}$. However in high risk group it was not so. In our study we found that CIN rate was high in patients having creatinine of $1.4-2 \mathrm{mg}$ group, which also had associated comorbidities. GFR is more sensitive risk predictor for CIN. As in our study, Creatinine value was in the range of 1.4 to $2.0 \mathrm{mg}$ in 158 cases, whereas according to GFR, 56 cases were having GFR range of $45-59$, that is low risk and 84 were in stage III that is at moderate risk for CIN. According to creatinine cutoff, CIN developed predominantly in group of patients who had creatinine range of $1.2-2 \mathrm{mg}$, but according to GFR criteria, all of them were having eGFR $<60$, that are at low to moderate risk. Thus eGFR would be the best risk predictor than creatinine.

\section{Conclusion}

As we observed in our study that the incidence of CIN increased with increase in amount of $\mathrm{CM}$ used, we recommend reducing amount $\mathrm{CM}$ during CECT in CKD patients with co-morbidities. As risk of CIN increases with increased amount of contrast media, one should try to reduce amount of contrast media and where ever possible alternate investigations like MRI should be advised. It is advisable to assess the risk of CIN on the basis of eGFR than creatinine level and one must check post contrast creatinine on $2^{\text {nd }}$ and $5^{\text {th }}$ day to assess the occurrence of CIN. We also recommend pre-contrast prophylaxis such as hydration therapy and $\mathrm{N}$ - acetyl cysteine administration to reduce the risk of development of CIN, especially in CKD patients with comorbidities. 
Conflict of interest statement- The authors declare that they have no conflict of interest in this research.

Acknowledgements- The authors are very grateful towards all the participants who took part in the study and for contributions from the Department of Radiodiaganosis and Imaging, Kasturba Medical College, Manipal, Manipal University and Department of Medical Imaging Technology, School of Allied Health Sciences, Manipal University.

Funding: Nil, Conflict of interest: None initiated,

Permission from IRB: Yes

\section{References}

1. Groell R, Doerfler O, Schaffler GJ, Habermann W. Contrast-enhanced helical CT of the head and neck: improved conspicuity of squamous cell carcinoma on delayed scans. AJR Am J Roentgenol. 2001 Jun;176(6): 1571-5. : doi/abs/10.2214/ajr.176.6.1761571

2. What are contrast materials and how do they work? Radiologyinfo.org:2014;1-8.

3. Mccullough PA, Bertrand ME, Brinker JA, Stacul F. A Meta-Analysis of the Renal Safety of Isosmolar Iodixanol Compared With Low-Osmolar Contrast Media. J Am Coll Cardiol. 2006 Aug 15;48(4):692-9. Epub 2006 Jul 24.2006;48(4). DOI: 10.1016/j.jacc. 2006.02.073

4. Choi D, Oh B, Lee M, Park Y. Renal Toxicity Evaluation and Comparison Between Visipaque (Iodixanol) and Hexabrix (Ioxaglate) in Patients With Renal Insufficiency Undergoing Coronary Angiography. J Am Coll Cardiol. 2006 Sep 5;48(5):924-30. Epub 2006 Aug 17. DOI:10.1016/j.jacc.2006.06.047

5. Aspelin P, Aubry P, Fransson S-G, Strasser R, Willenbrock R, Berg KJ, et al. Nephrotoxic effects in high-risk patients undergoing angiography. N Engl J Med [Internet]. 2003 Feb 6 [cited 2016 Jun 29];348 (6):491-9. DOI: 10.1056/NEJMoa021833
6. Trial ARD, Nephropathy C, Solomon RJ, Natarajan MK, Doucet S, Sharma SK, et al. Cardiac Angiography in Renally Impaired Patients (CARE) Study Patients With Chronic Kidney Disease. Circulation. 2007; 115:3189- 3196 http://dx.doi.org/10. 1161/ CIRCULATIONAHA. 106.671644

7. AR, Kuhn MJ, Chen N, Beek EJR Van, So GJ. The PREDICT study: a randomized double-blind comparison of contrast-induced nephropathy after lowor isoosmolar contrast agent exposure. AJR Am J Roentgenol. 2008 Jul;191(1):151-7. doi: 10.2214/AJR. 07.3370 .

8. Nguyen SA, Suranyi P, Ravenel JG,, Randall PK, Romano PB, Strom KA, Costello P, Schoepf UJ. Isoosmolality versus low-osmolality iodinated contrast medium at intravenous contrast-enhanced CT: effect on kidney function.Radiology. 2008 Jul;248(1):97-105. doi: 10.1148 /radiol. 2481071484. Epub 2008 May 15.

9. Thomsen HS1, Morcos SK.. Risk of contrastmedium-induced nephropathy in high-risk patients undergoing MDCT - A pooled analysis of two randomized trials. Eur Radiol. 2009 Apr; 19(4):891-7. doi: 10. 1007 /s00330-008-1206-4. Epub 2008 Nov 11.

10. Heinrich MC1, Häberle L, Müller V, Bautz W, Uder M. Nephrotoxicity of Iso-osmolar Nonionic Lowosmolar Contrast Controlled Trials, Radiology: 2009 Jan; 250(1):68-86. doi: 10.1148/radiol.25010 80833.

11. Hernández F, Mora L, García-tejada J, Velázquez M, Gómez-blázquez I, Bastante T, et al. Comparison of Iodixanol and Ioversol for the Prevention of ContrastInduced Nephropathy in Diabetic Patients After Coronary Angiography or Angioplasty. Rev Esp Cardiol. 2009 Dec;62(12):1373-80. DOI: http://dx.doi. org/10. 1016/j.rec.2016.05.033

\section{How to cite this article?}

Thomas Mathew, Dr Prakashini K, Rahul P Kotian. Identification of Contrast Induced Nephropathy (CIN) with intravenous iso-Osmolar contrast media in renal failure patients with and without additional comorbidities undergoing Computed Tomography. Int J Med Res Rev 2016;4(12):2108-2113.doi:10.17511 /ijmrr. 2016.i12.05. 